\title{
Numerical Analysis of a Radiation-Cooled Micro Arcjet Thruster
}

\author{
By Hiroyuki ОкАмото ${ }^{1)}$ and Michio Nishida ${ }^{2)}$ \\ 1) Astro Research Corporation, Yokohama, Japan \\ ${ }^{2)}$ Department of Aerospace Systems Engineering, Sojo University, Kumamoto, Japan
}

(Received November 8th, 2004)

\begin{abstract}
The flow field in a micro arcjet thruster using argon as propellant was analyzed numerically using the Direct Simulation Monte Carlo (DSMC) method. Subsonic inlet conditions were imposed to correctly simulate the actual flow inside the thruster. It was assumed that the input power was partitioned to propellant molecules to change their thermal velocities. Wall temperature was determined by the balance of conductive heat flux and radiative heat release. The primary purpose of the present work is to investigate the effects of the nozzle half-cone angle and input power on thruster performance characteristics. The results provide engineering direction to the micro arcjet thruster design.
\end{abstract}

Key Words: Arcjet Thruster, Micro Thruster, Electric Propulsion, DSMC

\author{
Nomenclatures \\ $C$ : thermal velocity vector \\ $c_{\text {in }}$ : incident molecule velocity at wall \\ $c_{\text {ref }}$ : reflected molecule velocity at wall \\ $F$ : thrust \\ $F_{\mathrm{N}}$ : number of molecules that simulated molecule repre- \\ sents \\ $I_{\text {sp }}:$ specific impulse \\ $\dot{m}$ : propellant mass flow rate \\ $m_{\mathrm{a}}:$ molecular mass \\ $N_{\mathrm{d}}$ : number of molecule in discharge section \\ $P_{\mathrm{w}}$ : input power \\ $R$ : gas constant \\ $\boldsymbol{R}_{3}$ : random vector \\ $T_{\mathrm{w}}$ : wall temperature \\ $\boldsymbol{U}$ : flow velocity vector \\ $V$ : flow velocity at inlet \\ $\eta$ : thrust efficiency \\ $\epsilon$ : input energy per molecule \\ $\tilde{\epsilon}$ : emissivity \\ $\sigma$ : Stephan-Boltzmann constant \\ $\rho$ : density
}

\section{Introduction}

Recently, much attention has been focused on small satellites. The ballistic coefficient of a small satellite is less than that of a normal satellite, so that the orbit altitude can be lower, leading to high-resolution remote-sensing data. Development of small satellites have been started for this reason. However, the amount of electric power generated by solar panels on satellites is comparatively small. Taking this limited condition into account, electric propulsion for small satellites is being developed. An arcjet thruster is one candidate for such electric propulsion. If arcjet thrusters

(C) 2006 The Japan Society for Aeronautical and Space Sciences are considered for the orbital station keeping of small satellites, they must be small. Moreover, since the input power to the arcjet thruster is small, the propellant flow rate must be small to raise the input energy density.

Two types of the arcjet thruster are being developed for use on small satellites: a pulsed arcjet thruster, ${ }^{1)}$ and a DC arcjet thruster. ${ }^{2)}$ The former uses a high-voltage pulsed-arc as heat source to avoid the difficulty of stabilizing a stationary DC arc. The later is basically the same as the conventional DC arcjet thruster on general satellites, and uses the stationary DC arc as a heat source.

The propellant flow rate in both these types of arcjet thrusters is very small, so the flow field inside the arcjet thruster must be treated as rarefied. The direct simulation Monte Carlo (DSMC) method ${ }^{3)}$ is adequate to solve rarefied gas dynamics problems. Boyd et al. ${ }^{4)}$ solved the flow field in the normal DC arcjet thruster using the DSMC method. The ionization-recombination reactions were the main purpose of their study, and the inlet condition was assumed to be supersonic.

In this work, the DSMC was applied to analyze the flow field inside a micro arcjet thruster in detail. Unlike the analysis of Boyd et al., ${ }^{4)}$ a subsonic condition is used as the inlet condition to simulate an actual micro arcjet thruster. The purpose is to reveal the flow characteristics inside a micro arcjet thruster to investigate the effect of nozzle expansion angle and input power on thrust performance.

\section{Flow Model and Numerical Calculation}

The following assumptions are made to simplify the analysis:

- The flow field is axisymmetric to simulate an actual arcjet thruster.

- The propellant is argon.

- The ionization-recombination reaction is neglected.

- Fully diffusive reflection at the nozzle wall is assumed. 


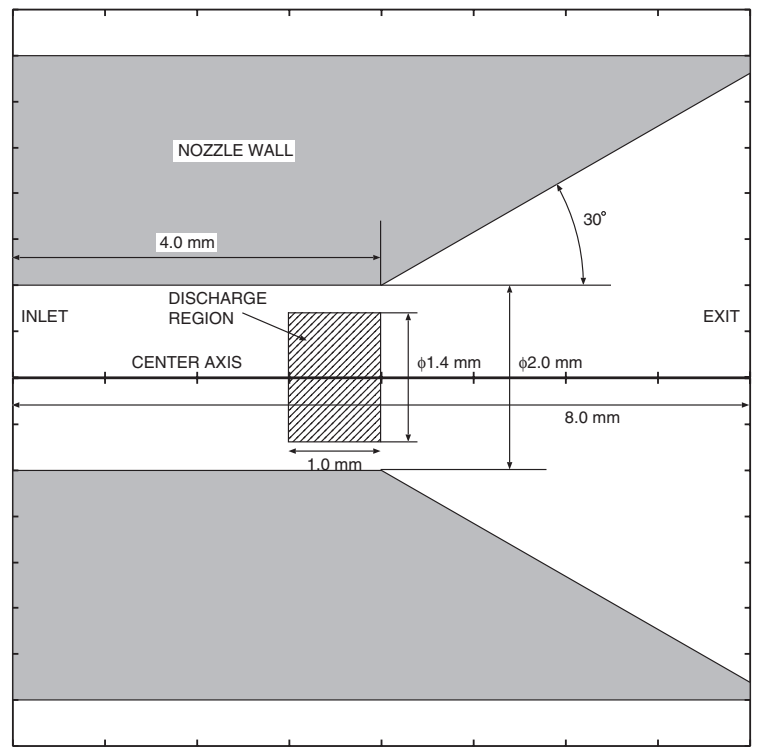

Fig. 1. Geometry of micro arcjet thruster.

- A variable hard sphere collision model is used.

- Arc power is injected to the discharge region with a specified volume.

Argon gas was chosen as the propellant because intermolecular collisions are simpler than between multi-atomic molecules, and, moreover the purpose of this work is to deduce basic knowledge about the flow field in the micro arcjet thruster. It is assumed that input energy is partitioned uniformly to molecules inside the specified volume.

The geometry of the present arcjet thruster is shown in Fig. 1. The constrictor is $2 \mathrm{~mm}$ in diameter and $4 \mathrm{~mm}$ in length. The nozzle expansion part is $4 \mathrm{~mm}$ in length, and its half cone angle is varied from $15^{\circ}$ to $60^{\circ}$ to investigate the effects of nozzle expansion angle on specific impulse and thrust efficiency. The propellant gas enters the left inlet and flows to the right exit through the arc discharge section. The propellant gas is arc-heated in the specified discharge volume, which is $1 \mathrm{~mm}$ in length and $1.4 \mathrm{~mm}$ in diameter, inside the constrictor. The diameter of the discharge section is chosen as 0.7 of the constrictor diameter because the arc radius in the conventional arcjet thruster is roughly 0.7 to 0.8 of the constrictor radius. ${ }^{5)}$ However, the geometry of the discharge section will affect the flow field in the arcjet thruster. Therefore, further investigations of this are necessary.

The arc power injected to the propellant gas heats up electrons first, and then the high electron energy is transferred to ions and neutral atoms through intermolecular collisions. In the present study, only argon atoms are considered, so the energy exchange between electrons and ions and between electrons and atoms should be included in the analysis. This process is a thermal nonequilibrium process. However, in the present analysis, the process of arc heating is assumed to be in thermal equilibrium. In other words, a one-fluid model is used to simplify the analysis. The total moment of the propellant molecules is not changed due to the energy injection. Therefore, the injected energy is considered used to just increase the thermal velocity of atoms. The molecule velocities are changed by the injected energy in this model.

The following numerical calculations are performed as follows:

$$
\begin{array}{r}
\frac{1}{2} m_{\mathrm{a}}\left\{\boldsymbol{C}_{0}+\boldsymbol{U}_{0}\right\}^{2}+\epsilon \\
=\frac{1}{2} m_{\mathrm{a}}\left\{\boldsymbol{C}_{1}+\boldsymbol{U}_{1}\right\}^{2}
\end{array}
$$

where, $m_{\mathrm{a}}$ is the molecular mass, $\boldsymbol{C}_{0}$ and $\boldsymbol{C}_{1}$ are the molecule thermal velocity vectors before and after energy injection, respectively, $\boldsymbol{U}_{0}$ and $\boldsymbol{U}_{1}$ are the flow velocity vectors before and after energy injection, respectively, and $\epsilon$ is the injected energy per molecule. Taking the average of both the leftand right-hand sides of Eq. (1) over the molecular velocities, we get

$$
\begin{gathered}
\frac{1}{2} m_{\mathrm{a}}\left\langle\left\{\boldsymbol{C}_{0}+\boldsymbol{U}_{0}\right\}^{2}\right\rangle+\langle\epsilon\rangle \\
=\frac{1}{2} m_{\mathrm{a}}\left\langle\left\{\boldsymbol{C}_{1}+\boldsymbol{U}_{1}\right\}^{2}\right\rangle
\end{gathered}
$$

where, \langle\rangle indicates the average over the molecular velocities. The flow velocity shall not be changed to fulfill the mass and moment conservation laws, and

$$
\langle\boldsymbol{C} \cdot \boldsymbol{U}\rangle=0
$$

So, the injected energy contributes only to the change of the thermal velocities. Consequently Eq. (2) can be written as

$$
\frac{1}{2} m_{\mathrm{a}}\left\langle C_{0}{ }^{2}\right\rangle+\langle\epsilon\rangle=\frac{1}{2} m_{\mathrm{a}}\left\langle C_{1}{ }^{2}\right\rangle
$$

It is assumed that a change of the velocity vector due to the energy injection is expressed by

$$
\boldsymbol{C}_{1}=\boldsymbol{C}_{0}+\alpha \boldsymbol{R}_{3}
$$

where, $\boldsymbol{R}_{3}$ is the random vector with random direction and length, and its mean length is unity. Since $\boldsymbol{C}_{0}$ and $\boldsymbol{R}_{3}$ are individual random vectors, the average inner product of both vectors are

$$
\left\langle\boldsymbol{C}_{0} \cdot \boldsymbol{R}_{3}\right\rangle=0
$$

Therefore, substituting Eq. (4) into Eq. (3), we get

$$
\begin{aligned}
& \frac{1}{2} m_{\mathrm{a}}\left\langle C_{0}{ }^{2}\right\rangle+\langle\epsilon\rangle \\
& \quad=\frac{1}{2} m_{\mathrm{a}}\left\langle C_{0}{ }^{2}\right\rangle+\frac{1}{2} m_{\mathrm{a}}\left\langle\alpha^{2}\right\rangle
\end{aligned}
$$

Equation (5) gives the following relation:

$$
\alpha=\sqrt{2\langle\epsilon\rangle / m_{\mathrm{a}}}
$$

Letting $N_{\mathrm{d}}$ and $P_{\mathrm{w}}$ be the number of molecules in the discharge section and the input power, respectively, the average energy injection per molecule $\langle\epsilon\rangle$ during $\Delta t$ is

$$
\langle\epsilon\rangle=\frac{P_{\mathrm{w}} \Delta t}{N_{\mathrm{d}}}
$$

Applying above result to molecules in the numerical cell, 
$\alpha_{\text {cell }}$ is expressed as

$$
\alpha_{\text {cell }}=\sqrt{2 \bar{\epsilon} / m_{\mathrm{a}}}
$$

and

$$
\bar{\epsilon}=\frac{P_{\mathrm{w}} \tau \Delta t}{F_{\mathrm{N}} \bar{N}_{\mathrm{d}}}
$$

where, $\Delta t$ is the time interval per step, $\tau$ is the number of sampling steps, $\bar{N}_{\mathrm{d}}$ is the accumulated number of simulated molecules in the discharge volume, and $F_{\mathrm{N}}$ is the real number of molecules that a simulated molecule represents. The number of simulated molecules is accumulated during the sampling intervals. In this study, $\tau$ was set to 10 , meaning the $\alpha_{\text {cell }}$ is recalculated every 10 steps, and $\alpha_{\text {cell }}$ is fixed during 10 steps. Asymptotic results are obtained by applying the above procedure.

The flow in the constrictor is subsonic, so large numbers of particle are required to simulate the flow field, which needs an extreme number of inter molecular collisions. Therefore, from the economics viewpoint, a smaller subsonic region is better for numerical simulation. However, subsonic inlet conditions are important in the flow field simulation of an arcjet thruster. Therefore, the inlet conditions are taken at the constrictor to reduce the subsonic region as much as possible. The diameter and length of the constrictor were taken to be $2 \mathrm{~mm}$ and $4 \mathrm{~mm}$, respectively. It is assumed that an arc strikes near the constrictor exit, so that a discharge section is as indicated in Fig. 1.

The inlet temperature and mass flow rate are specified to treat the subsonic inlet conditions correctly. The number of molecules reaching the inlet plane $N_{\mathrm{IN}}$ is given by ${ }^{6)}$

$$
N_{\mathrm{IN}}=n_{\mathrm{IN}} \sqrt{\frac{R T}{2 \pi}} K(V / \sqrt{2 R T})
$$

where, $n_{\mathrm{IN}}$ is the number density at the inlet, $V$ is the flow velocity at the inlet, $R$ is the gas constant and

$$
K(x)=\exp \left(-x^{2}\right)+\sqrt{\pi} x(1+\operatorname{erf} x) .
$$

Since the flow velocity cannot be fixed, the asymptotic process has been applied to determine the flow velocity. The subsonic inlet conditions were treated as follows:

1. Suppose inlet velocity.

2. Conduct numerical simulation until steady state solution obtained.

3. Calculate mass flow rate at inlet and exit.

4. Renew inlet velocity by calculated mass flow rate.

5. Repeat steps 1 to 4 until mass flow converges.

The nozzle wall temperature was assumed to be uniform and the heat influx to the wall was also assumed to be balanced with radiative heat release.

$$
\begin{aligned}
Q_{\mathrm{rad}} & =\tilde{\epsilon} \sigma T_{\mathrm{w}}{ }^{4} S_{\mathrm{rad}} \\
& =\frac{F_{\mathrm{N}} m_{\mathrm{a}}}{2} \frac{\sum c_{\mathrm{in}}{ }^{2}-\sum c_{\mathrm{ref}}{ }^{2}}{\Delta t}
\end{aligned}
$$

where, $Q_{\text {rad }}$ is the radiative heat flux, $T_{\mathrm{w}}$ is the nozzle wall temperature, $\tilde{\epsilon}$ is the emissivity, $\sigma$ is the Stephan-Boltzmann constant, $F_{\mathrm{N}}$ is the real number of molecules that a sample molecule represents, and $c_{\text {in }}$ and $c_{\text {ref }}$ are the incident and reflected molecule velocities at the wall, respectively.

The wall temperature was treated as follows:

1. Suppose nozzle wall temperature.

2. Conduct numerical simulation until steady state solution obtained.

3. Count income and reflected energy.

4. Modify wall temperature to meet Eq. (11).

5. Repeat steps 1 to 4 until Eq. (11) satisfied.

The DSMC method was used in this study. The arc-off flow fields were calculated at first until the flow properties, especially the mass flow rate, converged. Then, the arc-on flow fields were calculated using the arc-off results as an initial condition until the flow properties converged. The converged flow field properties were obtained by averaging the sampling of the simulation results.

\section{Results and Discussion}

Table 1 summarizes the conditions for the present analysis.

Figure 2 shows the simulation grids. Test runs were carried out to determine the grid resolution. As a result 40 grids in the flow direction and 10 grids in the radial direction were chosen for the present analysis.

In Figs. 3 to 7, computed results are shown for the nozzle half-cone angle of $30^{\circ}$, nozzle length of $8 \mathrm{~mm}$, propellant mass flow rate of $1.0 \mathrm{mg} / \mathrm{s}$ and arc power of $10 \mathrm{~W}$. Density contours of the flow fields for arc off and arc on are shown in Fig. 3. The temperature inside a satellite in space is assumed to be at $0^{\circ} \mathrm{C}$. Hence, the inlet and wall temperatures were set to $273.15 \mathrm{~K}$. It is apparent that the density contours are different at arc off and arc on. The inlet density of the archeated flow is about three times larger than that for arc

Table 1. Computational conditions.

\begin{tabular}{ll}
\hline Mass flow rate & $1.0 \mathrm{mg} / \mathrm{s}$ \\
Initial propellant temperature & $273.15 \mathrm{~K}$ \\
Knudsen number & $6 \times 10^{-3}$ \\
$\quad$ (based on constrictor radius) & \\
Input power & $0 \mathrm{~W}, 1 \mathrm{~W}$ \\
& and $10 \mathrm{~W}$ \\
Nozzle half-cone angle & $15^{\circ}$ to $60^{\circ}$ \\
\hline
\end{tabular}

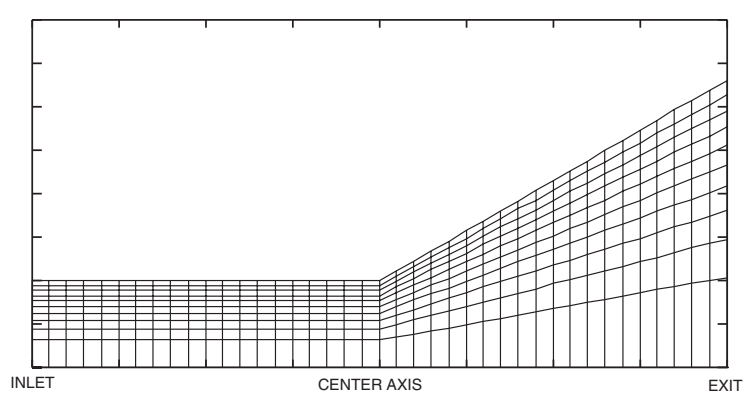

Fig. 2. $40 \times 10$ grids. 


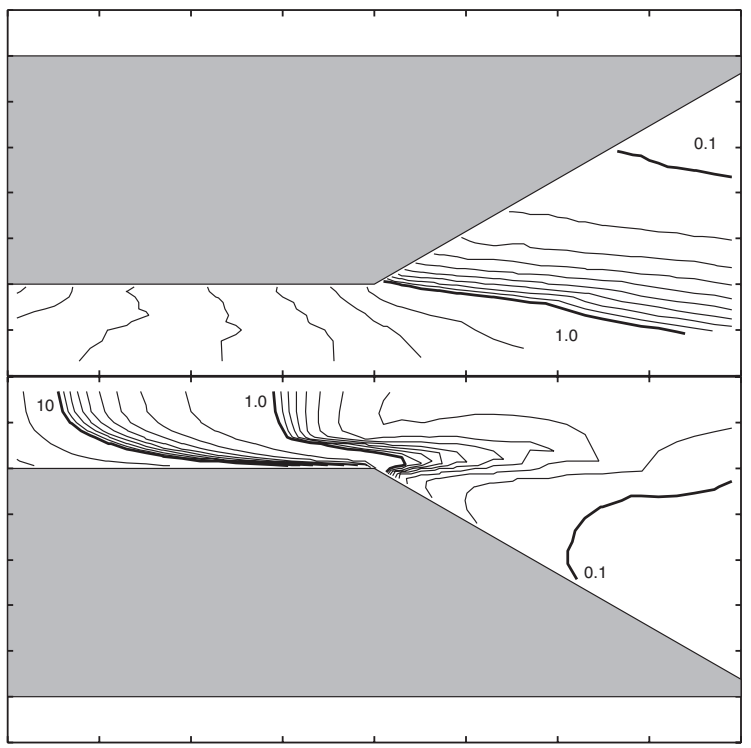

Fig. 3. Density contours $\left(\dot{m}=1.0 \mathrm{mg} / \mathrm{s}, \rho_{0}=2.96 \mathrm{~g} / \mathrm{m}^{3}\right.$, Upper: arc off, Lower: $\operatorname{arc}$ on $P_{\mathrm{w}}=10 \mathrm{~W}$ )

off, causing increase in the stagnation pressure.

A density gradient is seen at the inlet for arc on. Adding a convergent section upstream of the constrictor, test runs were conducted to investigate the effect of the inlet conditions on the downstream flow field. No significant difference from the present result was seen, and hence the numerical region was determined as the present one.

However, the density near the constrictor exit is more dilute than in the arc-off case. Also, the density gradient around the discharge section is steeper than in the arc-off flow. It can be seen that there is a high-density ring jet around the constrictor exit near nozzle wall. In other words, the density in the discharge section is more rapidly expanded than around the discharged section. The density gradient at the nozzle exit is seen to be smaller than in the arc-off case, which means that the input power is used for propellant acceleration.

Figure 4 shows the convergence history of the mass flow rate at the inlet and exit. The mass flow rate was different from the actual rate at the beginning of the simulation. However, the converged result was obtained as the simulation

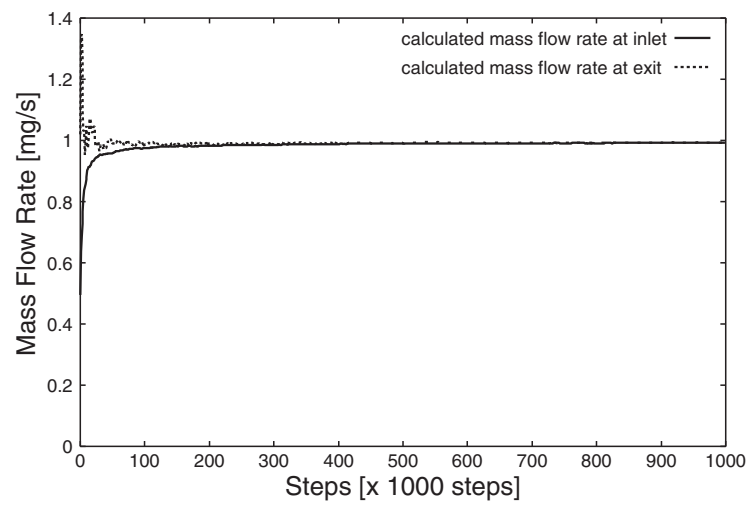

Fig. 4. Mass flow rate convergence $\left(\dot{m}=1.0 \mathrm{mg} / \mathrm{s}, P_{\mathrm{w}}=10 \mathrm{~W}\right)$.

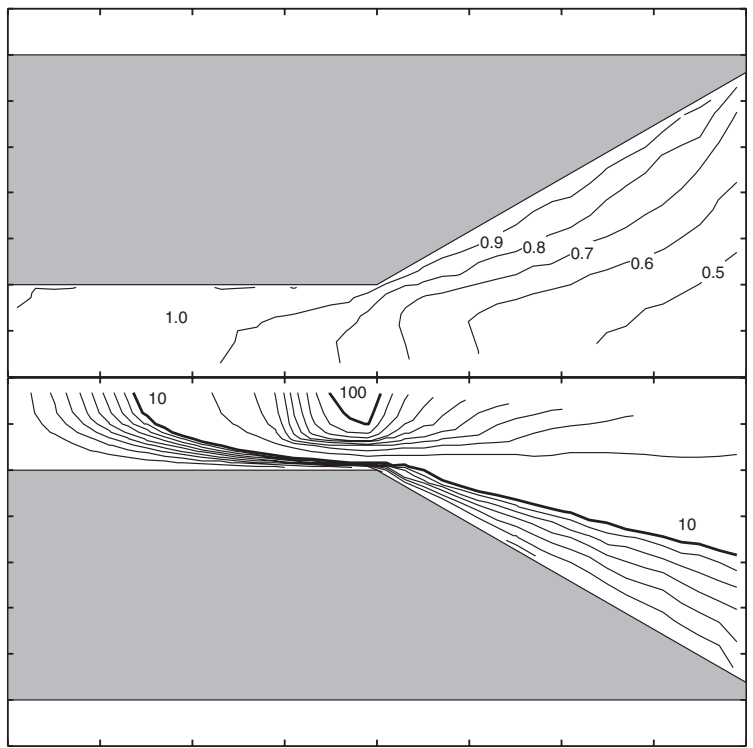

Fig. 5. Temperature contours $\left(\dot{m}=1.0 \mathrm{mg} / \mathrm{s}, T_{0}=273.15 \mathrm{~K}\right.$, Upper: arc off, Lower: $\operatorname{arc}$ on $P_{\mathrm{w}}=10 \mathrm{~W}$ ).

step goes by. This also shows that the simulated results are accurate in terms of mass conservation.

Figure 5 shows the temperature contours for arc on and off. It can be seen that the downstream temperature in the constrictor propagates upstream. The computed maximum temperature is about $20,000 \mathrm{~K}$. This temperature is not realistic. The real maximum temperature will be reduced owing to the ionization reaction of the propellant gas. The heat release due to radiation cooling is $4.3 \mathrm{~W}$ when the input power is $10 \mathrm{~W}$. Therefore, the radiation loss is $43 \%$ of the input power. The conventional arcjet thruster has around $50 \%$ of radiation loss.

Ionization due to arc-heating is not described here, and in actual thrusters, the ionization consumes part of the arc power. Therefore, the present input power of $10 \mathrm{~W}$ may be considered as the residual power after ionization energy loss. When the ionization-recombination reaction is applied, the energy balance is different from the present balance, and the radiation loss ratio to the total power will be smaller than the present result. Therefore, the micro arcjet thruster can have less radiation loss than the conventional arcjet thruster.

Comparing the density and temperature contours, the temperature in the ring jet is colder than the core flow around center. Figure 6 shows the density distribution and

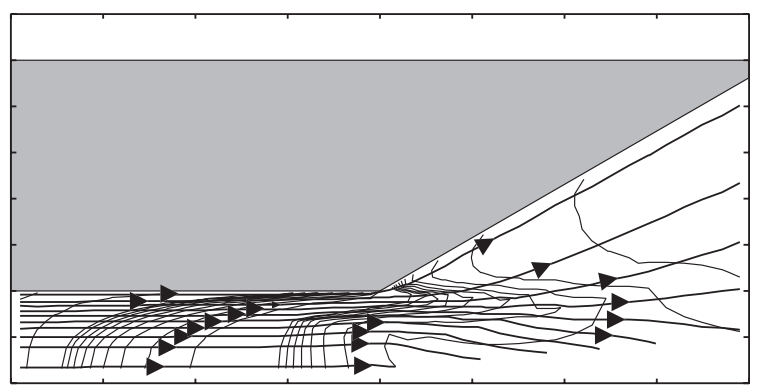

Fig. 6. Density contours and stream lines $\left(\dot{m}=1.0 \mathrm{mg} / \mathrm{s}, P_{\mathrm{w}}=10 \mathrm{~W}\right)$. 


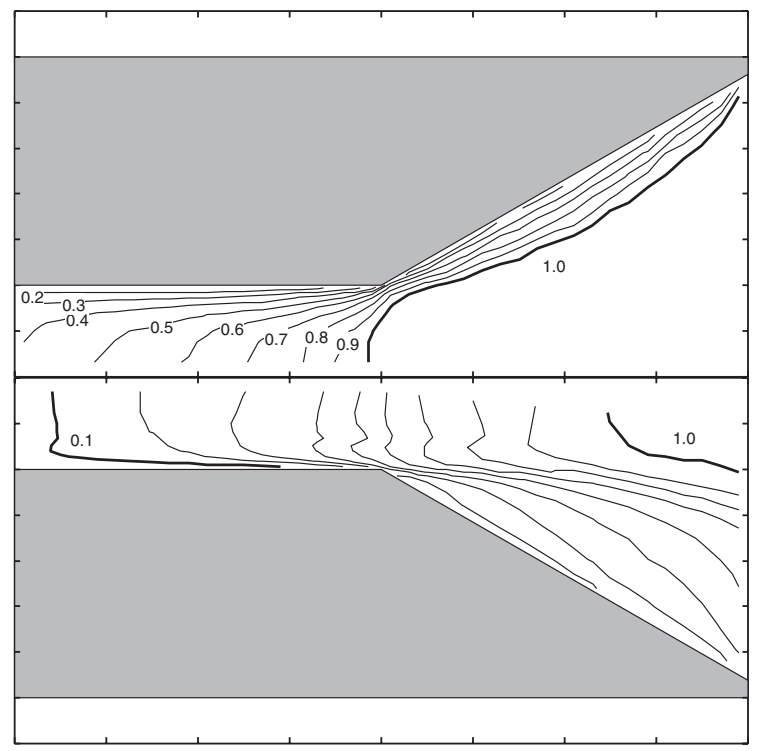

Fig. 7. Mach number contours $(\dot{m}=1.0 \mathrm{mg} / \mathrm{s}$, Upper: arc off, Lower: arc on, $\left.P_{\mathrm{w}}=10 \mathrm{~W}\right)$.

the stream lines of the discharged flow field. The ring jet is expanded to both nozzle wall and center axis. Therefore, the core flow is cooled by the ring jet.

Figure 7 shows the Mach number contours of the arc-off and arc-on flow field. The sonic line is located away from the constrictor exit in the discharged flow. The sonic condition is not only a function of nozzle geometry but also heating location. The temperature downstream of the constrictor remains high, so it requires a similar length to the constrictor diameter for a flow to become supersonic.

The performance characteristics obtained by the computation are summarized in Table 2.

Thrust $F$, specific impulse $I_{\text {sp }}$ and thrust efficiency $\eta$ are calculated as follows:

$$
\begin{gathered}
F=\int \rho u^{2}+p d A \\
I_{\mathrm{sp}}=F / \dot{m} \\
\eta=\frac{F^{2} / 2 \dot{m}}{\dot{m} C_{\mathrm{p}} T_{0}+P_{\mathrm{w}}}
\end{gathered}
$$

where, $\rho$ is the density, $u$ is the axial velocity component, and $p$ is the pressure, respectively, at the nozzle exit. $C_{\mathrm{p}}$ is the specific heat at constant pressure of the propellant and $T_{0}$ is the propellant temperature at the inlet. Figure 8 shows the specific impulse and thrust efficiency as a function of input power. The specific impulse increases with input power.

Table 2. Computed performance.

\begin{tabular}{ll}
\hline Mass flow rate (specified) & $1.0 \mathrm{mg} / \mathrm{s}$ \\
Inlet temperature (specified) & $273.15 \mathrm{~K}$ \\
Input power (specified) & $10 \mathrm{~W}$ \\
Thrust (computed) & $2.19 \mathrm{mN}$ \\
Specific impulse (computed) & $223 \mathrm{~s}$ \\
Thrust efficiency (computed) & $23.6 \%$ \\
\hline
\end{tabular}

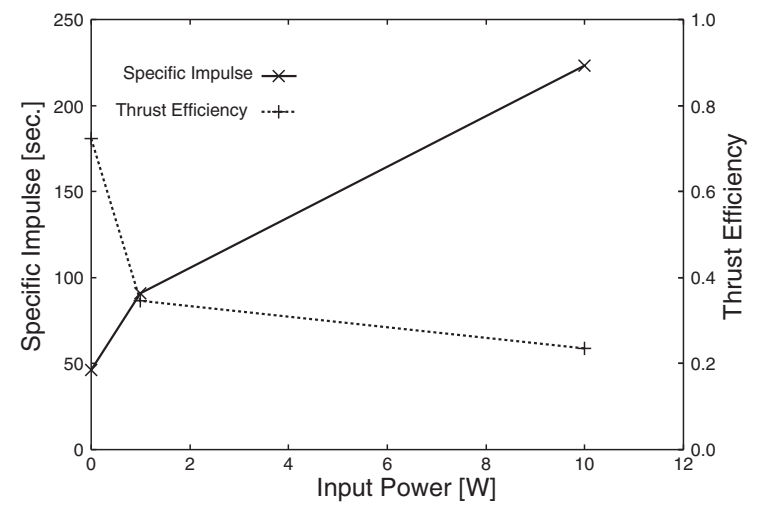

Fig. 8. Specific impulse and thrust efficiency vs input power.

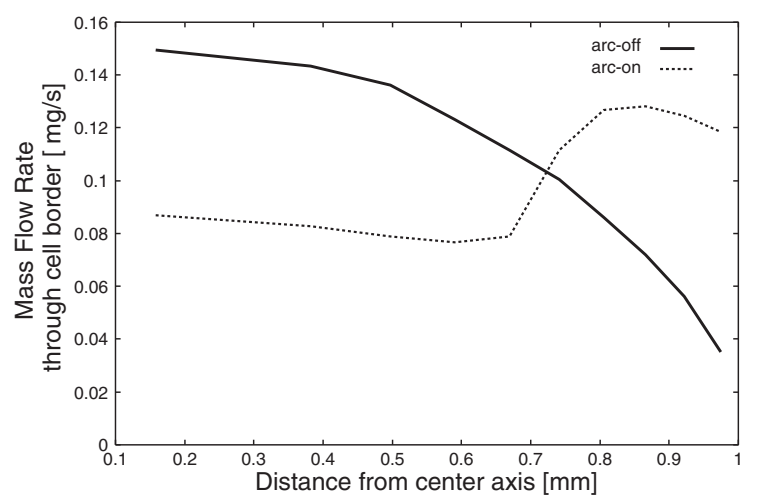

Fig. 9. Mass flow rate through cell boundary.

Conversely, thrust efficiency decreases as the input power increases. This feature is similar to that of a conventional arcjet thruster.

The radial profile of the mass flow through the cell boundary at the constrictor exit is shown in Fig. 9. It can be seen that the mass flow through the heating section is less than that through the outside of it. The flow outside the heating section forms a ring jet. This ring jet is colder than the core flow, so that the core heats the ring jet. Perhaps this ring jet reduces the momentum of the core flow which has a higher velocity than the ring jet. The existence of ions and electrons is not considered in this study. Even if ions and electrons are included in the analysis, the energy transferred by these ions and electrons will be less than that of neutral molecules, because the degree of ionization is generally very low in arcjet thrusters, typically $10^{-5}$. Therefore, the present flowfield result does not change when ions and electrons are considered. Since the ring jet is formed near the nozzle wall, the wall geometry may affect the arcjet thruster performance.

Simulations were also conducted for nozzle half-cone angles of $15^{\circ}, 30^{\circ}, 45^{\circ}$ and $60^{\circ}$. Figure 10 shows the specific impulse and thrust efficiency as a function of the nozzle half-cone angle. The input power, constrictor diameter and nozzle exit diameter were fixed for all nozzle half-cone angles. The nozzle length was changed according to the nozzle half-cone angle. The specific impulse and thrust efficiency increase with half-cone angle. There is an optimum halfcone angle when the flow is a continuum. If the half-cone 


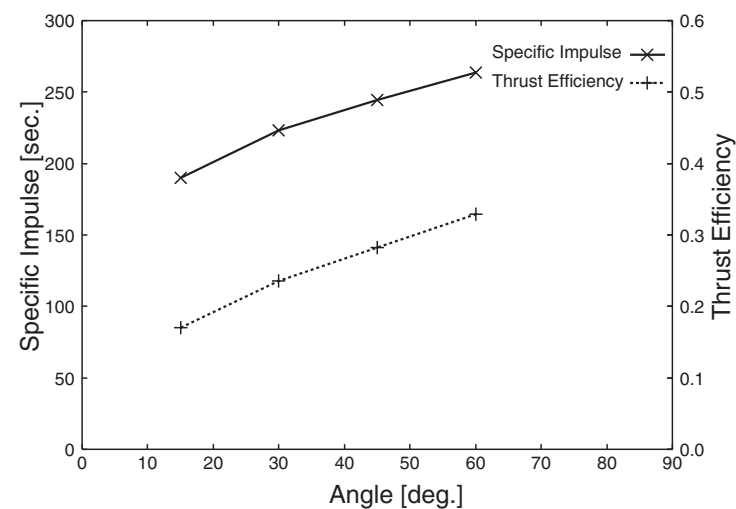

Fig. 10. Specific impulse and thrust efficiency vs nozzle wall angle ( $\dot{m}=$ $\left.1.0 \mathrm{mg} / \mathrm{s}, P_{\mathrm{w}}=10 \mathrm{~W}\right)$.

angle is larger than optimum, the flow separates from the nozzle wall and a shock wave appears. The nozzle wall influences the radiation cooling. Therefore, the loss of input power is affected by nozzle length. Generally speaking, a smaller nozzle length is better from the energy viewpoint. However, in this study, a larger half-cone angle is better even if the flow separates from the nozzle wall. However, the plume widens as nozzle half-cone angle increases. Therefore, the nozzle half-cone angle shall also be considered from the plume viewpoint. An additional plume shield outside of the arcjet thruster may be a candidate engineering solution.

\section{Conclusions}

The major difference between the micro DC arcjet thruster and the conventional one is the very low flow rate. Although the Knudsen number is high at the subsonic inlet in the micro DC arcjet thruster, the flow will be rarefied at the throat exit. Therefore, numerical analysis of the internal flow of the micro DC arcjet thruster requires the direct sim- ulation Monte Carlo method.

The conclusions of this study are summarized as follows:

1. The flow of a micro DC arcjet thruster was simulated by using the direct simulation Monte Carlo method.

2. The sonic line appears far downstream of the constrictor exit at arc on.

3. The ring jet appears at the constrictor exit. This ring jet has most of the flow rate.

4. The nozzle wall half-cone angle affects the arcjet thruster performance. The larger the wall half-cone angle, the better the specific impulse and thrust efficiency.

\section{Acknowledgments}

This research was partially supported by Grant-in-Aids for Scientific Research, No. 15106013, sponsored by the Ministry of Education, Culture, Sports, Science and Technology, Japan.

\section{References}

1) Willmes, G. F. and Burton, R. L.: Low-Power Helium Pulsed Arcjet, J. Propul. Power, 15 (1999), pp. 440-446.

2) Ashiya, H., Horisawa, H. and Kimura, I.: Discharge Characteristics of a Very Low-Power Arcjet, 1G3, the 46th Space Sciences and Technology Conference, Tokyo, 2002 (in Japanese).

3) Bird, G. A.: Molecular Gas Dynamics and Direct Simulation of Gas Flows, Oxford University Press, New York, 1994

4) Boyd, I. D.: Extensive Validation of Monte Carlo Method for Hydrogen Arcjet, J. Propul. Power, 13 (1997), pp. 775-782.

5) Martinez-Sanchez, M.: Simplified Analysis of Arcjet Operation, Lecture 11-12, 16.522 Space Propulsion, Aeronautics and Astronautics, Masachusetts Institute of Technology, 2004; URL http://ocw.mit. edu/NR/rdonlyres/Aeronautics-and-Astronautics/16-522Spring2004/ 030CF8D9-FFA9-41C3-B430-7E49311E6D6D/0/lecture11_12.pdf

6) Serikov, V. V. and Nanbu, K.: Methodological Aspects of MCDS Approach as Applied to 3-D Flow in the Sputtering Chamber, The Reports of the Institute of Fluid Science, Tohoku University, Vol. 6, 1994, pp. 43-72. 